\title{
Effect of surface roughness on sliding friction of micron-sized glass beads
}

\author{
Jan MEYER, Regina FUCHS, Thorsten STAEDLER*, Xin JIANG \\ Chair of Surface and Materials Technology, University of Siegen, 57068 Siegen, Germany \\ Received: 04 December 2013 / Revised: 03 March 2014 / Accepted: 17 March 2014 \\ (C) The author(s) 2014. This article is published with open access at Springerlink.com
}

\begin{abstract}
In order to understand the contact phenomena of micron-sized particles, which have a tremendous impact on a variety of applications in industry and technology, direct access to the loads as well as the displacements accompanying such contacts are mandatory. Typical particle ensembles show a size variation ranging from the nanometer to the tenths of micron scale. Especially the contact behavior of particles featuring radii of several up to several tenths of microns is scarcely studied as these particles are typically too large for atomic force microscopy (AFM) based approaches and too small for conventional macroscopic testing setups. In this work a nanoindenter based approach is introduced to gain insight into the contact mechanics of micron-sized glass beads sliding on rough silicon surfaces at various constant low normal loads. The results are analyzed by a simple modified Coulomb friction law, as well as Hertz, JKR, and DMT contact theory.
\end{abstract}

Keywords: sliding friction; roughness; colloid probe technique; particle technology; nanoindentation

\section{Introduction}

The contact mechanics of particle ensembles critically depend on the individual contact of particles as well as particles and boundaries/walls. Consequently, in order to predict or model the behavior of such ensembles direct experimental access to the parameters describing the contact of individual particles is required $[1,2]$.

In the context of nanoparticles as well as particles featuring radii up to a couple of microns scanning probe microscopy represents today's most prominent contact method to sample the interaction of individual particles as well as particles and walls, i.e., the colloid probe technique $[3,4]$. Unfortunately, this approach typically is limited with respect to load, particle size, and particle motion. Macroscopic techniques, on the other hand, fail to handle individual micron-sized particles with the required resolution in load and

* Corresponding author: Thorsten STAEDLER.

E-mail: thorsten.staedler@uni-siegen.de

A preliminary version of this work was presented at the $3 \mathrm{rd}$ International Symposium on Tribology of IFToMM, Luleå, Sweden, 2013. displacement. In Refs. [5,6] we demonstrated the potential of a nanoindentation based colloid probe approach. This strategy allowed for the assessment of sliding, rolling and torsional friction of individual micron sized spherical particles.

One of the crucial parameters strongly influencing the lateral force required to slide a particle over a surface is the real contact area between the two. Both particle and surfaces are not perfectly smooth and typically feature surface asperities on the nanometer scale. An increasing surface roughness tends to increase the mean separation between two interacting bodies, which results in a decrease of adhesion as well as affect the sliding friction coefficient. Investigations concerned with tribological phenomena have been carried out for atomically smooth surfaces $[7,8]$ as well as surfaces featuring different degrees of roughness [9]. Aside from a potentially strong adhesive contribution [10], in all cases Amontons' law is valid. However, a variation in friction coefficient can be measured and several models [11,12] have been developed to correlate this variation with the corresponding variation in surface roughness. The most prominent theories have been proposed by Bowden 
and Tabor [11] and Greenwood et al. [13, 14] who suggested that the real contact area increased with applied load due to surface roughness. The results are in accordance with Amontons' law if plastic deformation of surface asperities was assumed.

Based on these findings, in the present work, we address the question of real contact area by varying the roughness as well as the applied normal force between the contact partners, i.e., spherical probe and surface. Particles are slid over a surface in a linear fashion repeatedly under constant load. The relationship between lateral force and applied normal load as well as number of cycles is analyzed. A modified Coulomb friction law [7] as well as the assumption of a Hertz contact [15], and contacts based on JKR [16] and DMT [17] theory is utilized to reproduce the behavior. The results are discussed and compared with supplementary adhesion measurements.

\section{Experimental details}

As the majority of experimental details are already reported in Ref. [5] we will only briefly summarize them here. The cyclic sliding as well as the analysis based on the various contact models, however, is described in more depth as this represents the focus of the present work and has not been shown elsewhere.

\subsection{Preparation of particle probes}

In order to prepare particle probes with well-defined surface conditions we selected borosilicate glass beads provided by Duke Scientific that feature nominal particle radii of $10 \mu \mathrm{m}$ (Duke Standards 9020). These spherical particles were fixed by means of photosensitive acrylate-based adhesive glue (DIC Europe $\mathrm{GmbH})$ to a cube corner diamond indenter tip (Hysitron Inc.), which featured a cavity at its apex prepared by focused ion beam (FIB) (FEI Helios 600).

\subsection{Preparation of silicon surfaces}

The choice of contact partners for the particle probes was motivated by analogous consideration. P-doped silicon wafers ((Si100), Siegert Wafer $\mathrm{GmbH}$ ) were chosen as they offered an ease of availability along with low initial surface roughness. The surface topography was subsequently changed by a micro wave plasma assisted chemical vapor deposition based etch process. Micro wave power determined the final roughness of the etched surfaces. Following the plasma treatment, the modified samples were stored under ambient conditions for two weeks, allowing the formation of a thin natural oxide layer.

\subsection{Characterization of roughness and adhesion}

The roughness of the Si surfaces as well as the apex of the particle probes was measured utilizing an atomic force microscopy (AFM) (PSIA XE-100) equipped with commercially available tips (ACTA, AppNano) featuring tip radii below $10 \mathrm{~nm}$ in non- contact mode.

AFM (Asylum Research MFP-3DTM AFM) based adhesion measurements were carried out with the borosilicate particles mentioned above set up as conventional colloidal probes. These probes were fashioned by means of gluing (Araldite $10 \mathrm{~min}, 2$ components, Epoxy) the borosilicate glass spheres $(10 \mu \mathrm{m}$ radius $)$ onto tipless cantilevers (NSC12, MikroMasch). Forcedistance curves were measured using a force volume (force map) method.

\subsection{Measuring the lateral force of a sliding contact}

All sliding experiments as well as the adhesion experiments mentioned above have been carried out at room temperature (RT) and $30 \pm 5 \%$ relative humidity (RH). In general, the particle probes were mounted to a nanoindenter setup (Hysitron Inc.), which was utilized to carry out the measurements. Two sets of experimental data were acquired. In the first set, lateral force data was collected in a friction loop fashion. The beads were slid over a surface length of $2 \mu \mathrm{m}$ at a constant normal load and a speed of $1 \mu \mathrm{m} / \mathrm{s}$. Normal loads of $3,10,30$, and $100 \mu \mathrm{N}$ were applied with the normal load being feedback controlled, respectively.

The second data set was also performed in a friction loop manner. This time, however, ten consecutive loops at a constant normal load were executed. Scratch length was changed to $10 \mu \mathrm{m}$. Here, normal loads of $1,3,6,10,30,60$, and $100 \mu \mathrm{N}$ were utilized, respectively.

The corresponding absolute value of lateral force for each test was evaluated by a lateral displacement sensitive averaging of the difference in measured 
lateral force for forward and backward movement divided by two. In order to avoid any artifact originating from a change in the movement direction only the central $50 \%$ of the friction loop was taken into account.

\subsection{Analysis of the lateral force data}

The lateral force data collected as described in section 2.4 were analyzed in two different approaches. The first, simply assumed that the measured lateral force $F$ is directly proportional to the applied normal load $L$ modified by a potential adhesive load component $L_{\text {adh }}$ with the proportionality factor being the friction coefficient $\mu$, see Eq. (1). Such linear relationship is often described as modified Coulomb friction [7].

$$
F=\mu\left(L+L_{\text {adh }}\right)
$$

The second approach was based on the idea that, in a predominantly elastic contact, the lateral force is presumably given by Eq. (2):

$$
F=\tau A=\tau \pi a^{2}
$$

Here $\tau$ represents a critical shear strength per unit area and $A$ the contact area with a being the corresponding contact radius, respectively. The latter can be estimated by consulting some well-established contact models. For simplicities sake we took a simple Hertz contact as well as the JKR and DMT contact theory into consideration. Following a nomenclature proposed by Carpick et al. [18] Table 1 summarizes the corresponding contact radii. Here $R$ denotes the radius of the particle probe and $\gamma$ the work of adhesion between surface and particle probe, respectively.

Table 1 Contact radii given by the corresponding contact models.

\begin{tabular}{ll}
\hline Model & Contact radius \\
Hertz & $a=\left(\frac{L R}{K}\right)^{1 / 3}$ \\
JKR & $a=\left(\frac{1+\sqrt{1-\frac{L}{-(2 / 3) \pi \gamma R}}}{2}\right)^{2 / 3}\left(\frac{6 \pi \gamma R^{2}}{K}\right)^{1 / 3}$ \\
DMT & $a=\left(1-\frac{L}{-2 \pi \gamma R}\right)^{1 / 3}\left(\frac{2 \pi \gamma R^{2}}{K}\right)^{1 / 3}$
\end{tabular}

$K$, see Eq. (6), represents the reduced contact modulus times $4 / 3$. In this equation $v$ and $E$ designate the Poisson ratio and Young's modulus of surface and particle probe, respectively.

$$
K=\left(\frac{4}{3}\right)\left(\frac{\left(1-v_{\text {surface }}^{2}\right)}{E_{\text {surface }}}+\frac{\left(1-v_{\text {particle }}^{2}\right)}{E_{\text {particle }}}\right)^{-1}
$$

Table 2 gives an overview of the values of individual parameters used for all evaluations throughout this study.

Table 2 Values of parameters used throughout this study.

\begin{tabular}{lcc}
\hline \multicolumn{1}{c}{ Parameter } & Silicon $(100)$ & Particle \\
\hline Young's modulus, $E$ & $179 \mathrm{GPa}$ & $71 \mathrm{GPa}$ \\
Poisson ratio, $v$ & 0.25 & 0.17 \\
Radius, $R$ & - & $8.5 \mu \mathrm{m}$ \\
\hline
\end{tabular}

\section{Results and discussion}

\subsection{Characterization of particle probes and $\mathrm{Si}$ surfaces}

Prior to testing, all particle probes were carefully inspected by high resolution scanning electron microscope (SEM, ZEISS Ultra 55) to ensure a clean contact area. Additionally, all probes used in this study showed no hysteresis in load displacement curves monitored during normal loading into fused silica up to loads a hundred times larger than those used during the sliding experiments reported here. This indicates that the glue will not cause any artifacts in the load signals gathered during tribological testing. The particle probes showed a root mean square (RMS) roughness of $0.7 \pm 0.1 \mathrm{~nm}$, which is in perfect agreement with the findings reported by van Zwol et al. [19]. The results for roughness and particle adhesion of the Si surfaces are given in Table 3.

Table 3 Summary of roughness and adhesion results of the different Si surfaces.

\begin{tabular}{lcc}
\hline \multicolumn{1}{c}{ Si surface } & RMS $(\mathrm{nm})$ & Adhesion force $(\mu \mathrm{N})$ \\
\hline As received & $0.3 \pm 0.1$ & $3.2 \pm 0.3$ \\
Etched $(1,600 \mathrm{~W})$ & $1.5 \pm 0.2$ & $2.7 \pm 0.3$ \\
Etched $(1,800 \mathrm{~W})$ & $2.7 \pm 0.4$ & $1.9 \pm 0.3$ \\
\hline
\end{tabular}


The roughness of these surfaces increased with increasing microwave power of the etching process. At the same time the height distribution itself shows Gaussian characteristics indicating a randomly rough surface in all three cases. The adhesion, however, decreases with increasing surface roughness. The latter agrees well with results reported by Liu et al. [20].

\subsection{Correlation between lateral forces during sliding and surface roughness}

In Refs. $[5,6]$ we already reported results of an analysis of our first data set. Assuming the lateral forces show a linear relationship, see Eq. (1), with respect to the applied normal loads friction coefficients as well as adhesive load components according to Table 4 are acquired. In this case, the adhesive load component represents the intercept with the abscissa for a fit of the whole normal load regime.

Two aspects of these results are somewhat surprising: (i) Etched Si surfaces show a significantly increased friction coefficient compared to the as received $\mathrm{Si}$ surface and (ii) $L_{\text {adh }}$ does not agree with the measured AFM based adhesion forces, see Table 3. However, the resulting friction coefficient of the as received $\mathrm{Si}(100)$ wafer in contact with the spherical probe of $0.23 \pm 0.05$ is in good agreement with the work of $\mathrm{Yu}$ et al. [12], who probed the transition from stick to slip for the contact of micron-sized silica spheres on a

Table 4 Results of analyzing data set one by assuming a modified Coulomb relationship.

\begin{tabular}{lcc}
\hline \multicolumn{1}{c}{ Si surface } & $\mu$ & $L_{\text {adh }}(\mu \mathrm{N})$ \\
\hline As received & $0.23 \pm 0.05$ & $12 \pm 1.2$ \\
Etched $(1,600 \mathrm{~W})$ & $0.53 \pm 0.05$ & $4.5 \pm 0.6$ \\
Etched $(1,800 \mathrm{~W})$ & $0.65 \pm 0.14$ & $7.8 \pm 1.2$ \\
\hline
\end{tabular}

$\mathrm{Si}(100)$ wafer depending on the relative humidity, as well as findings by Zhang et al. [21], who studied the sliding friction of silica colloidal probes on microsphere-patterned silicon surfaces. Further agreement is found with reports by Maharaj et al. [22] and Quintanilla et al. [23].

In the following we will try to address the inconsistency of the measured adhesion force (AFM) with respect to the one obtained from a linear fit to the lateral force data. In order to do so it is instructive to take a closer look at the fits of the first data set with respect to modified Coulomb friction Eq. (1) as well as the other contact models mentioned in section 2.5 (see Fig. 1). In case of the etched Si surfaces the modified Coulomb relationship is a good approximation in describing the correlation between lateral force and applied normal force. Here the contact between particle and surface can be viewed as a true multiasperity contact resembling contact situations on a macroscopic level. In case of the contact between the as received $\mathrm{Si}$ surface and the glass bead, however, no distinction with respect to one specific contact model can be made. All of the four attempts show equally good agreement with the data set.

In order to resolve this issue the same analysis was repeated with lateral forces obtained from the first friction loop of the second experimental data set. Comparing these results with the ones for the first experimental data set a striking agreement between both series is found. However, in case of the as received Si surface in contact with the particle probe, the additional data points of the new data set are helpful to shed some light on the details of the sliding contact at low applied normal loads (see Fig. 2). The corresponding non-linear behavior with respect to the full normal load regime, which shows more
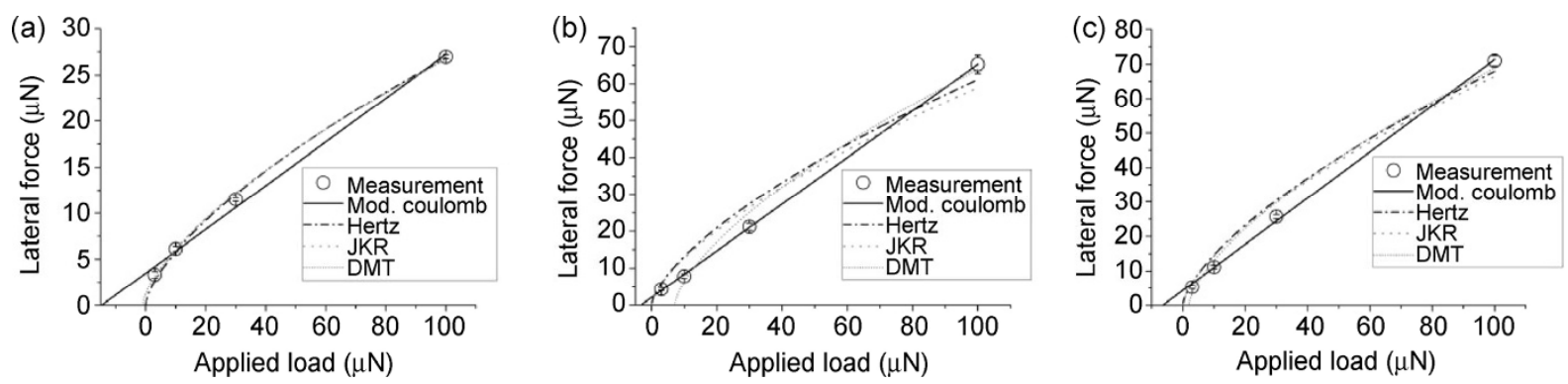

Fig. 1 Comparison between experimental data (set one) and a linear interrelationship (mod. Coulomb) as well as other contact models, for sliding contact between bead and surface: (a) as received, (b) etched at $1600 \mathrm{~W}$, and (c) etched at $1800 \mathrm{~W}$. 


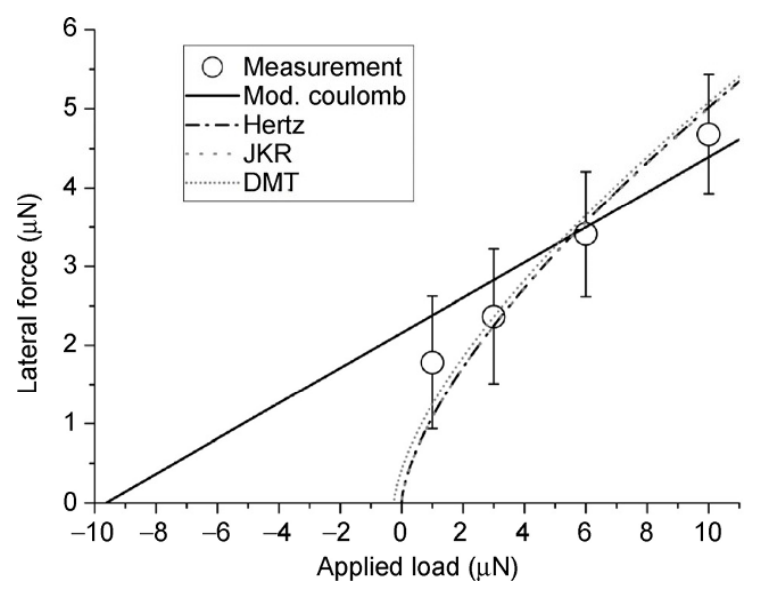

Fig. 2 Low load regime of the sliding contact between as received Si surface and glass bead along with corresponding fitting results.

resemblance to a single asperity contact, is given in Fig. 3(a)).

By critically analyzing Fig. 2 one observes that neither the modified Coulomb relationship nor the other contact models, which were all fit to the whole data range up to $100 \mu \mathrm{N}$ applied load, agree well with the measured data below $10 \mu \mathrm{N}$. In fact, a rather linear trend is observed for the measurement in the applied load regime between 1 and $10 \mu \mathrm{N}$, which, however, features a steeper slope, i.e., higher friction coefficient, compared to the one obtained for linearly fitting the complete range of applied normal load. At the same time the intercept of the low load trend with the axes of applied load would lead to a better agreement with $L_{\text {adh }}$ measured by AFM. Unfortunately, in such a case, it is not possible to predict the appropriate load regime resulting in the adhesion between the contacting partners a priori. A true clarification on this issue, that ultimately also affects the contact of rougher partners, would, however, require access to measurements at negative applied loads. The corresponding modifications of the nanoindentation equipment are work in progress and will be reported elsewhere.

After commenting on the question of the affective adhesive force component during sliding, we still have to consider the issue of the relatively high friction coefficients in case of the etched Si surfaces. In Refs. $[5,6]$ we reasoned that plastic deformation of surface asperities might be responsible for this phenomenon. A quick check on this proposition by means of characterization of area wear marks generated at very low loads $(<10 \mu \mathrm{N})$ confirmed the occurrence of plastic deformation. In these experiments the topography of the contacting surfaces showed a reduced roughness while no obvious wear debris was formed. Consequently, we decided to gain detailed insight into the key mechanisms by analyzing the time dependency of lateral forces gathered during consecutive friction loops at a constant applied load over a fixed linear surface region. The corresponding results and discussion will be presented in the following paragraph.

\subsection{Evolution of lateral forces of a multi-cycle friction loop sliding contact}

If the high friction coefficients obtained for etched surfaces are results of plasticity occurring on an asperity level during the sliding contact, a significant change in lateral forces after the first cycle of a multicycle sliding contact at constant applied load is
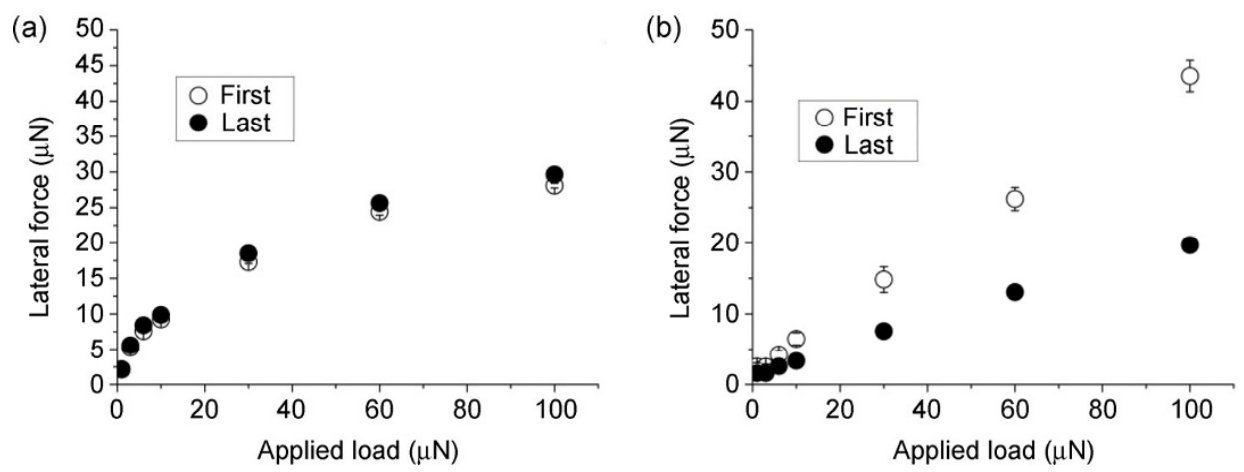

Fig. 3 Lateral forces measured for the first and last (tenths) cycle under constant normal loads: (a) As received Si and (b) Si surface etched in a process that featured $1,800 \mathrm{~W}$ microwave power. 
expected. Indeed such changes could be observed for the new set of experimental data. Figure 3 shows representative sets of lateral forces detected during the first as well as the tenths (last) cycle of sliding over as received and etched Si surfaces, respectively. Again the relationship between lateral load and applied load in case of the as received Si surface features a clear non-linear correlation. This observation stays true even after ten cycles. The rougher surface, on the other hand, displays a linear interrelationship between lateral force and normal force in case of the first as well as the last cycle. The absolute values of detected lateral forces, however, behave significantly different for the two surfaces. While the lateral forces slightly increase for the as received surface, a dramatic drop in lateral forces is noted for the rougher surface between the first and tenths cycle.

In order to study any potential dependency of this phenomena with respect to applied normal load the respective changes for all individual applied normal loads and all three tested surfaces are given in Fig. 4. As already noticed in Fig. 3 a clear difference between the as received surface and the rougher surfaces is observed. The change in detected lateral force for a given surface, however, is apparently independent of applied normal load within the load regime studied here. The biggest drop in lateral forces is seen for the roughest sample tested. In this case, the lateral forces

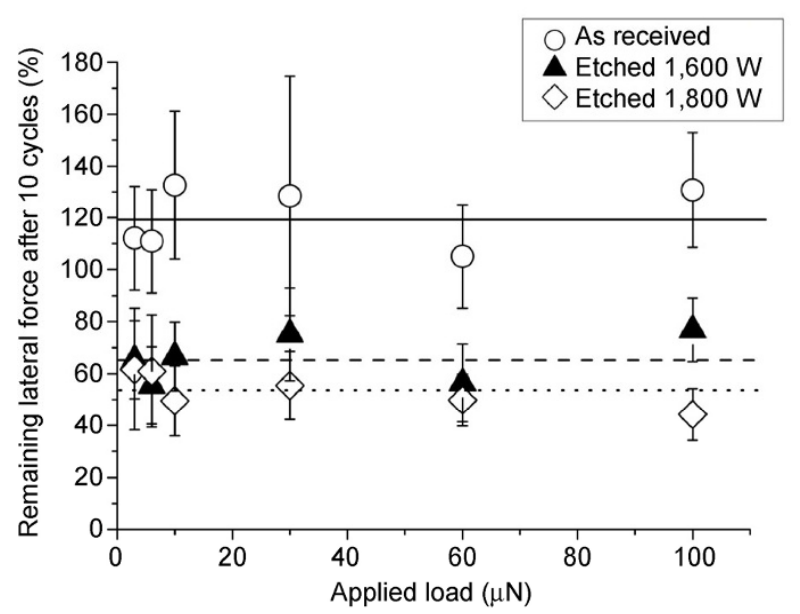

Fig. 4 Change in detected lateral forces between first and tenths cycle for all tested surfaces and applied normal loads. The horizontal lines represent the average values of a given surface for all applied normal loads, respectively. decrease to about $50 \%$ of their initial (first cycle) value during the course of ten cycles.

Finally, Fig. 5 displays a representative set of data collected at $60 \mu \mathrm{N}$ constant applied load revealing details of the evolution of the lateral force during the ten consecutive cycles of sliding. This plot holds a wealth of information. It turns out, in case of the etched surfaces, that the results of the first cycle are clearly dominated by plastic deformation. After a few cycles the effect of any plastic events in the contact zone is significantly decreased. The etched surface featuring the lower RMS roughness reaches this kind of equilibrium surface state earlier compared to the rougher Si surface. The latter shows a slight final decreasing trend in lateral force that might very well continue beyond the tenths cycle. Even for the as received surface the occurrence of plastic events during the first cycle cannot be excluded. The small increase in lateral force after the first cycle might reflect on a slightly lower contact roughness, which leads in turn to an increased real contact area and, therefore, an increased lateral force. Staying true to this line of thought, the fact that the absolute values of lateral forces for the rough surfaces after ten cycles are well below the one of the as received surface implies that the final surface states of these rougher surfaces feature a smaller real contact area compared to the as received one. The details of the evolution of the topography in the sliding track will be a complex function based on the applied normal load, mechanical

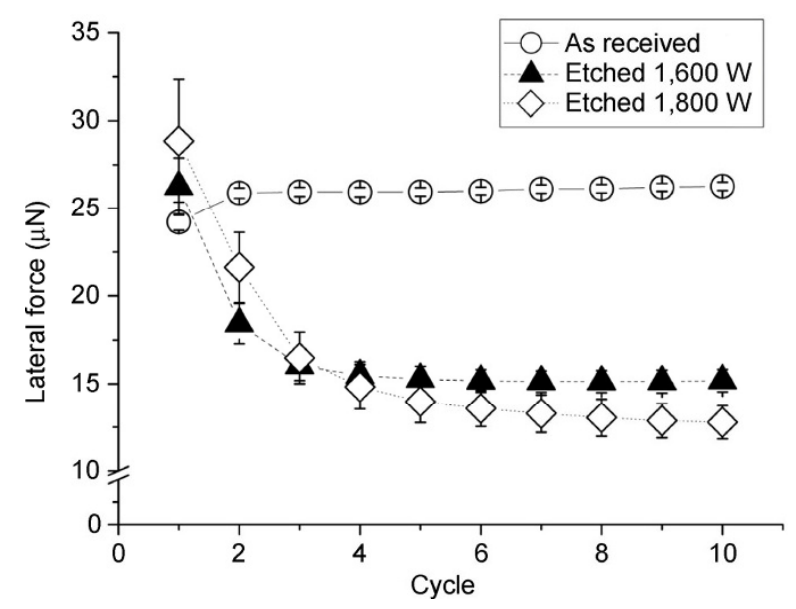

Fig. 5 Detailed evolution of lateral forces during the ten cycles of sliding at $60 \mu \mathrm{N}$ constant applied normal load for all three surfaces tested in this study. 
properties of the contact partners, number of cycles, and initial roughness as well as initial local curvature and local slope of surface asperities of both contact partners, respectively. In principle it is assumed that the topography of the contacting partners changes until the local contact pressure on an asperity level finally falls below the strength of the corresponding materials. Studying this evolution in more detail is quite a challenge as high resolution in surface characterization as well as high reproducibility of positioning of the glass bead is required in order to provide meaningful information. Dedicated efforts are in progress to derive appropriate strategies to succeed in this endeavor.

In order to check on the effect of any tribochemical reaction on the detected lateral forces, as reported in Ref. [12], the sliding experiments on the Si surface etched at $1,600 \mathrm{~W}$ were repeated under a dry $(2.4 \%$ $\mathrm{RH})$ nitrogen atmosphere. The comparison between the previous results and the once under dry condition are shown in Fig. 6. Both experiments feature a very similar trend, indicating that with respect to the contact conditions studied here plastic deformation of individual surface asperities might play a more significant role compared to tribochemical reactions. It is, given the current set of experimental data, however, not possible to rule out the influence of tribochemistry on the results.

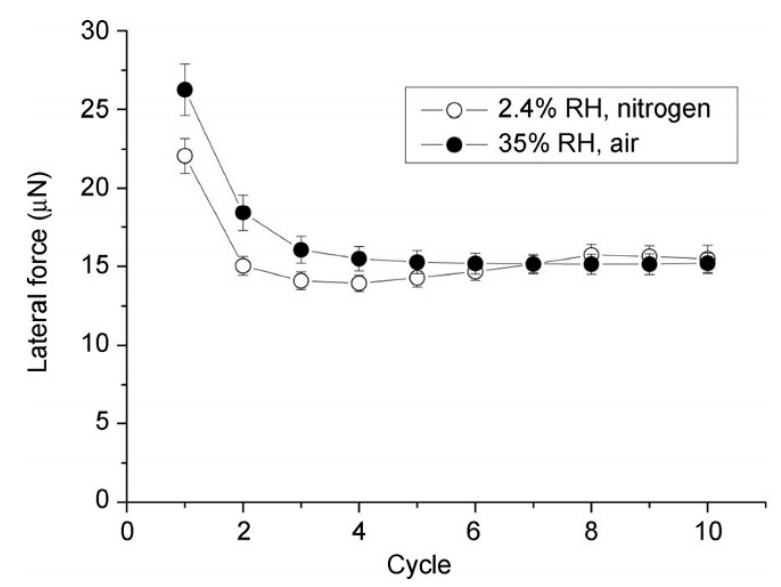

Fig. 6 Comparison of the evolution of lateral force for the contact between a glass bead and the $\mathrm{Si}$ surface etched at $1,600 \mathrm{~W}$ for two different atmospheric conditions. The tests were carried out at $60 \mu \mathrm{N}$ normal load and 35\% RH in air as well as $2.4 \% \mathrm{RH}$ in nitrogen, respectively.

\section{Conclusion}

In this work we extended the concept of the AFM based colloid probe technique to a nanoindenter setup. By doing so it was possible to study sliding friction between borosilicate glass beads featuring nominal radii of $10 \mu \mathrm{m}$ and Si surfaces. The roughness of the Si surfaces was varied by means of a plasma etching process.

The relationship between lateral forces and the corresponding applied normal loads in a load regime between 1 and $100 \mu \mathrm{N}$ was studied. The lateral forces showed a linear trend with respect to the applied normal loads for the etched, i.e., rough and surfaces, which was analyzed by a modified Coulomb friction law. In case of the smooth as received $\mathrm{Si}$ wafer surface, a non-linear interrelationship between lateral force and applied normal force was observed, which agreed well with a Hertz based contact behavior. Therefore, it is deduced, that it was possible to observe a transition from an ideal Hertz-like contact in case of the smooth $\mathrm{Si}$ wafer to a multi-asperity contact in case of the rougher Si surfaces. A persistent discrepancy between adhesion forces derived from AFM based test and the ones concluded based on the analysis by a modified Coulomb friction law or even JKR and DMT contact models was observed. Further insight will only be possible once the test setup is able to carry out tests at negative applied normal loads. The corresponding modifications of the equipment are work in progress.

The relative high initial sliding friction coefficients of $0.53 \pm 0.05$ and $0.65 \pm 0.14$ for Si surfaces etched at a plasma power of $1,600 \mathrm{~W}$ and $1,800 \mathrm{~W}$, respectively, could be related to a significant plastic deformation of surface asperities during the initial friction loop. In fact, analyzing the tenth cycle of the Si surfaces etched at $1,800 \mathrm{~W}$ by a modified Coulomb friction law yields a friction coefficient of $0.19 \pm 0.05$. This friction coefficient is actually even slightly smaller compared to the one received for the initial contact cycle of the as received $\mathrm{Si}$ wafer surface, i.e., $0.23 \pm$ 0.05 . Potentially this small drop in friction coefficient is related to a smaller final real contact area between the glass bead and the etched surface after ten cycles compared to the real contact area between bead and smooth Si wafer surface. 


\section{Acknowledgement}

The authors would like to thank Dr. H. Zhuang, University of Siegen, for his support in preparing rough silicon surfaces and the German Research Foundation (DFG) for financial support under grant Nos. STA $1021 / 1-1$ and STA 1021/1-2. The work is carried out within the framework of the Key Research Program (SPP 1486) "Particle in Contact".

Open Access: This article is distributed under the terms of the Creative Commons Attribution License which permits any use, distribution, and reproduction in any medium, provided the original author(s) and source are credited.

\section{References}

[1] Dominik C, Tielens A G G M. Resistance to rolling in the adhesive contact of 2 elastic spheres. Philos Mag A 72(3): 783-803 (1995)

[2] Tomas J. Adhesion of ultrafine particles-Energy absorption at contact. Chem Eng Sci 62(21): 5925-5939 (2007)

[3] Ducker W A, Senden T J, Pashley R M. Direct measurement of colloidal forces using an atomic force microscope. Nature 353(6341): 239-241 (1991)

[4] Butt H J. Measuring electrostatic, Vanderwaals, and hydration forces in electrolyte-solutions with an atomic force microscope. Biophys J 60(6): 1438-1444 (1991)

[5] Fuchs R, Meyer J, Staedler T, Jiang X. Sliding and rolling of individual micrometre sized glass particles on rough silicon surfaces. Tribology-Materials, Surfaces \& Interfaces 7(2): 103-107 (2013)

[6] Fuchs R, Weinhart T, Meyer J, Zhuang H, Staedler T, Jiang $\mathrm{X}$, Luding S. Rolling, sliding and torsion of micron-sized silica particles: Experimental, numerical and theoretical analysis. Granular Matter: 1-17 (2014)

[7] Gao J P, Luedtke W D, Gourdon D, Ruths M, Israelachvili J N, Landman U. Frictional forces and Amontons' law: From the molecular to the macroscopic scale. J Phys Chem B 108(11): 3410-3425 (2004)

[8] Ruan J A, Bhushan B. Atomic-scale and microscale friction studies of graphite and diamond using friction force microscopy.
J Appl Phys 76(9): 5022-5035 (1994)

[9] Koinkar V N, Bhushan B. Effect of scan size and surface roughness on microscale friction measurements. J Appl Phys 81(6): 2472-2479 (1997)

[10] Otsuki M, Matsukawa H. Systematic breakdown of Amontons' law of friction for an elastic object locally obeying Amontons' law. Sci Rep-Uk 3: 1596 (2013)

[11] Bowden F P, Tabor D. Friction lubrication and wear-A survey of work during last decade. Brit J Appl Phys 17(12): 1521 (1966)

[12] Yu J X, Chen L, Qian L M, Song D L, Cai Y. Investigation of humidity-dependent nanotribology behaviors of $\operatorname{Si}\left(\begin{array}{ll}1 & 0\end{array}\right.$ $0) / \mathrm{SiO}_{2}$ pair moving from stick to slip. Appl Surf Sci 265: 192-200 (2013)

[13] Greenwoo J A, Williams J B. Contact of nominally flat surfaces. Proc R Soc Lon Ser-A 295(1442): 300-319 (1966)

[14] Greenwoo J A. Constriction resistance and real area of contact. Brit J Appl Phys 17(12): 1621 (1966)

[15] Hertz H. Über die Berührung fester elastischer Körper. $J$ Für Die Reine U Angew Math 1882: 156-171 (1882)

[16] Johnson K L, Kendall K, Roberts A D. Surface energy and contact of elastic solids. Proc R Soc Lon Ser-A 324(1558): 301-313 (1971)

[17] Derjaguin B V, Muller V M, Toporov Y P. Effect of contact deformations on adhesion of particles. $J$ Colloid Interf Sci 53(2): 314-326 (1975)

[18] Carpick R W, Ogletree D F, Salmeron M. A general equation for fitting contact area and friction vs load measurements. $J$ Colloid Interf Sci 211(2): 395-400 (1999)

[19] van Zwol P J, Palasantzas G, Van De Schootbrugge M, De Hosson J T M, Craig V S J. Roughness of microspheres for force measurements. Langmuir 24(14): 7528-7531 (2008)

[20] Liu D L, Martin J, Burnham N A. Optimal roughness for minimal adhesion. Appl Phys Lett 91(4): 043107 (2007)

[21] Zhang X L, Zhong X H, Meng X, Yi G W, Jia J H. Adhesion and friction studies of nano-textured surfaces produced by self-assembling $\mathrm{Au}$ nanoparticles on silicon wafers. Tribol Lett 46(1): 65-73 (2012)

[22] Maharaj D, Bhushan B. Effect of spherical Au nanoparticles on nanofriction and wear reduction in dry and liquid environments. Beilstein J Nanotech 3: 759-772 (2012)

[23] Quintanilla M A S, Goddard D T. Lateral force microscopy with micrometer-sized particles: Effect of wear on adhesion and friction. Wear 268(1-2): 277-286 (2010) 


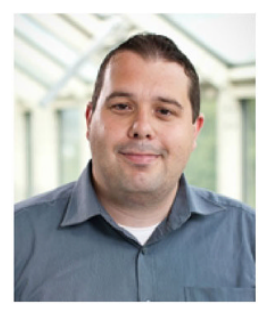

Jan MEYER. He received his Master degree in Chemistry in 2010 from the University Siegen, Germany. Till date, he carries out his $\mathrm{PhD}$ studies at the Institute of Materials

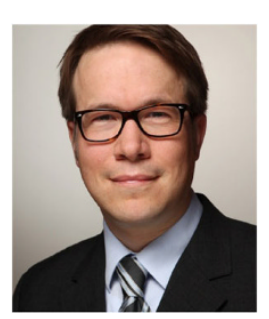

Thorsten STAEDLER. He received his BS degree in Physics from University of Hamburg, Germany, in 1994. Subsequently, he obtained his MS and PhD degrees in Physics from the Technical University of Braunschweig, Germany, in 1998 and 2001, respectively. After spending two years as
Engineering at the University Siegen, Germany. His research interests include particle technology and tribological phenomena of individual particles in corresponding ensembles of micron-sized granular media.

Postdoctoral Associate at Cornell University, Ithaca (NY), USA, he took the position as head of the analytical section of the Institute of Materials Engineering at the University Siegen, Germany. His research areas cover the mechanical and tribological characterization of nanostructured materials as well as thin film technology in general. 